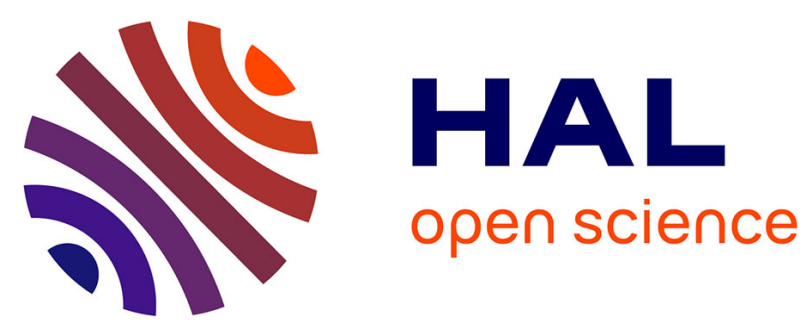

\title{
Improving Awareness for 3D Virtual Collaboration by Embedding the Features of Users' Physical Environments and by Augmenting Interaction Tools with Cognitive Feedback Cues
}

Thierry Duval, Thi Thuong Huyen Nguyen, Cédric Fleury, Alain Chauffaut, Georges Dumont, Valérie Gouranton

\section{To cite this version:}

Thierry Duval, Thi Thuong Huyen Nguyen, Cédric Fleury, Alain Chauffaut, Georges Dumont, et al.. Improving Awareness for 3D Virtual Collaboration by Embedding the Features of Users' Physical Environments and by Augmenting Interaction Tools with Cognitive Feedback Cues. Journal on Multimodal User Interfaces, 2014, 8 (2), pp.187-197. 10.1007/s12193-013-0134-z · hal-00908402

\section{HAL Id: hal-00908402 https://hal.inria.fr/hal-00908402}

Submitted on 22 Feb 2014

HAL is a multi-disciplinary open access archive for the deposit and dissemination of scientific research documents, whether they are published or not. The documents may come from teaching and research institutions in France or abroad, or from public or private research centers.
L'archive ouverte pluridisciplinaire HAL, est destinée au dépôt et à la diffusion de documents scientifiques de niveau recherche, publiés ou non, émanant des établissements d'enseignement et de recherche français ou étrangers, des laboratoires publics ou privés. 


\title{
Improving Awareness for 3D Virtual Collaboration by Embedding the Features of Users' Physical Environments and by Augmenting Interaction Tools with Cognitive Feedback Cues
}

\author{
Thierry Duval - T. T. Huyen Nguyen · Cédric Fleury · Alain Chauffaut • \\ Georges Dumont · Valérie Gouranton
}

Date of receipt: 6 June 2013. Date of acceptance: 22 November 2013

\begin{abstract}
The feeling of presence is essential for efficient interaction within Virtual Environments (VEs). When a user is fully immersed within a VE through a large immersive display system, his/her feeling of presence can be altered because of disturbing interactions with his/her physical environment, such as collision with hardware parts of the system or loss of tracking. This alteration can be avoided by taking into account the physical features of the user as well as those of the system hardware and embedding them in the VE. Moreover, the 3D abstract representation of these physical features can also be useful for collaboration between distant users because they can make a user aware of the physical limitations of the others he/she is collaborating with. In this paper we present how we use the
\end{abstract}

Thierry Duval

Université de Rennes 1, UEB, IRISA, UMR CNRS 6074,

France

E-mail: thierry.duval@irisa.fr

Huyen Nguyen

INRIA Rennes Bretagne-Atlantique, IRISA, UMR CNRS 6074, France

E-mail: thi-thuong-huyen.nguyen@inria.fr

Cédric Fleury

LRI, Université Paris-Sud, UMR CNRS 8623, France

E-mail: cedric.fleury@lri.fr

Alain Chauffaut

INRIA Rennes Bretagne-Atlantique, IRISA, UMR CNRS 6074, France

E-mail: alain.chauffaut@inria.fr

Georges Dumont

ENS Cachan antenne de Bretagne, UEB, IRISA, UMR CNRS 6074, France

E-mail: georges.dumont@irisa.fr

Valérie Gouranton

INSA de Rennes, UEB, IRISA, UMR CNRS 6074, France

E-mail: valerie.gouranton@irisa.fr
Immersive Interactive Virtual Cabin (IIVC) model to obtain this virtual representation of the user's physical environment and we illustrate how this representation can be used in a collaborative navigation task in a VE. We also present how we can add 3D representations of $2 \mathrm{D}$ interaction tools in order to cope with asymmetrical collaborative configurations, providing 3D cues for a user to understand the actions of the others even if he/she is not fully immersed in the shared VE. Last, we briefly explain how we plan to enhance 3D interaction and collaboration by embedding a symbolic 3D user representation that will give $3 \mathrm{D}$ information about his/her posture.

Keywords 3D Collaborative Virtual Environments, Awareness of Collaboration, Perception of the Physical workspaces of the users

\section{Introduction}

A 3D Virtual Environment (3D VE) is a virtual environment where $3 \mathrm{D}$ objects are displayed to a user. A user of such an environment is involved in a perception/action loop [41], and the success of his/her interactions contributes to his/her feeling of presence in the virtual environment [46]. Usually he/she can interact with this virtual environment through dedicated input devices. Chris Hand [26] proposed three categories of interactions: navigation (the interaction with the user's viewpoint), interaction with the virtual objects of the virtual environment (object selection, object manipulation), and application control (interaction with 3D widgets in order to change some parameters of the virtual environment). This is very similar to the four categories proposed by Bowman et al. [7] where the interaction 
with the objects of the virtual world is explicitly decomposed into selection and manipulation. Many efficient interaction techniques have been developed in this area in the past decades [8]. In addition, due to new 3D input devices and 3D displays becoming widely available for everyone, research in new 3D user interfaces is more relevant than ever [6]. When an interactive $3 \mathrm{D}$ Virtual Environment is deployed upon an immersive display system, such as a CAVE ${ }^{\mathrm{TM}}$ [13], a Head-Mounted Display (HMD), a workbench, or simply a large screen, we talk about using Virtual Reality techniques in order to explore this 3D Virtual Environment and interact with it.

A 3D Collaborative Virtual Environment (3D CVE) is an interactive $3 \mathrm{D}$ virtual environment where several local or distant users can join to share a collaborative interaction experience. It can be considered as a Cognitive Infocommunication system [3] that enables Intra-cognitive communication between users through representation-sharing and representation-bridging information. It can also be used for Inter-cognitive communication between users and (possibly remote) information. Such examples of Cognitive Infocommunication systems are presented in [25].

Moreover, 3D CVEs intend to make the users not just remotely communicate, but rather really interact together by sharing interactions in the $3 \mathrm{D}$ virtual environment. These interactions may happen on distinct objects, on different parts of a same object, or even on the same part (at the same time) of a shared virtual object [31].

Object manipulation is a fundamental task of $3 \mathrm{D}$ interaction in Virtual Reality (VR), and collaborative manipulation of virtual objects by multiple users is a very promising area [5]. Collaborative manipulation of objects is indeed necessary in many different applications of VR such as virtual prototyping, training simulations or assembly and maintenance simulations [40]. In such virtual collaborative tasks, all the users should participate naturally and efficiently to the motion applied to the object manipulated in the VE [24][28]. Another common use of $3 \mathrm{D}$ CVE is virtual navigation: collaborative visits (museums, cultural heritage, architectural/urban project reviews) or collaborative games (cars races).

In this paper section 2 presents the state-of-the-art on providing awareness to users of VEs. Section 3 recalls the IIVC concepts and how they can be applied, while sections 4 and 6 illustrate these concepts through an example of design and one example of implementation. Section 5 presents how users can be provided 3D cues to help them understand the actions of others even if they are not fully immersed in the shared virtual en- vironment. Finally, section 7 concludes the paper and gives an outline of future trends within this topic.

\section{Awareness in Virtual Environments}

Embedding the user's motion workspace into a virtual environment offers the user an intuitive way to navigate by moving his/her own body. It also makes it possible to manage problems induced by the fact that the virtual world is often larger than this real workspace. For example, the 3DM graphical modeler [9] enables a user to move on a "magic carpet" which represents the boundaries of the tracking area, he/she can perform real movements on the "magic carpet" to intuitively perform interactions. For long-distance navigation, he/she can also drive the "magic carpet" into the virtual world with a specific tool. For natural walking in virtual worlds within a restricted workspace, the "Magic Barrier Tape" [12] displays the boundaries of the physical workspace as a virtual barrier tape. It informs the user about the boundaries of his/her walking workspace defined by the tracking area or the display devices. Moreover, even if they do not display the user's motion workspace in the virtual environment, previous works about natural walking also have to consider these workspaces in order to prevent the user from colliding with the real environment or leaving the tracking area [39][45].

Within collaborative virtual environments, understanding issues may occur to users whose viewpoints are different [23]. Even if they can see the avatar of each other user, its position and its orientation in the virtual world as in CALVIN [29], users have difficulties perceiving what the others see, and more generally what they are doing and what they can do. To overcome these perception problems, Fraser et al. [23] explicitly outlined each user's view frustum using a wireframe model. By extension, the spatial model of interaction proposed by [4] can be seen as an interesting approach to describe users' multi-sensory perception. This spatial model defines sensory focus and nimbus for each user. The focus corresponds to the area in which a user has a sensory perception of other users or of virtual objects. The nimbus corresponds to the area in which the others have a sensory perception of this user. Moreover, users carry their focus and nimbus while they move in the virtual world.

To conclude, modeling users' physical environment improves user presence by matching the virtual world with the real world and by providing an environment safe from collisions or tracking problems. However, existing solutions do not deal with the representation of physical devices in the virtual environment, and they 
can neither describe the spatial relationships between these physical devices, nor model the users' physical workspace associated to each device. Other solutions describe the organization of users' physical environment by a hierarchy of coordinate systems and introduce the notion of workspace, but they do not consider the physical workspaces of a user as explicit $3 \mathrm{D}$ volumes. So the notion of workspace introduced by Mulder et al. [32] must be generalized to all the sensory workspaces and to various devices. This is why we need a generic model that enables VR developers to embed the users' physical environment into the VE when designing new applications, especially collaborative ones. We are presenting here an extended version of our research results previously published in [18].

\section{The IIVC Model}

We have proposed the Immersive Interactive Virtual Cabin (IIVC) [19] in order to cope with the problems mentioned above. The IIVC is a generic solution that considers the users' physical environment during the VR software design, its deployment and its use. This solution provides a high-level model to describe, configure and modify the users' physical workspace organization, whatever immersive devices are used.

We propose to model the users' physical environment as a structured hierarchy of virtual workspaces. Here we will focus mainly on the motion workspace (the area w a user can move his/her body), the visual workspace (what the user can see through and around a display device) and the interaction workspace (the area where a user can interact). We call stage the reference workspace of our hierarchy of workspaces that depicts the real-world spatial relationships between these workspaces.

\subsection{The IIVC Concept}

The IIVC can be defined as an abstraction of the users' physical environment in the virtual world. It enables developers to implement their VR software without considering the physical devices to be used. For example, developers only have to manage position, orientation and scale of each user's IIVC when they develop navigation techniques. In a second step, each IIVC is configured with the features of each user's physical devices (size, shape, hierarchy of workspaces). The IIVC is based on three main components: the workspace, the stage, and the conveyor:

- A workspace is a 3D space, defined by its position, orientation and size. Most of the time it is defined

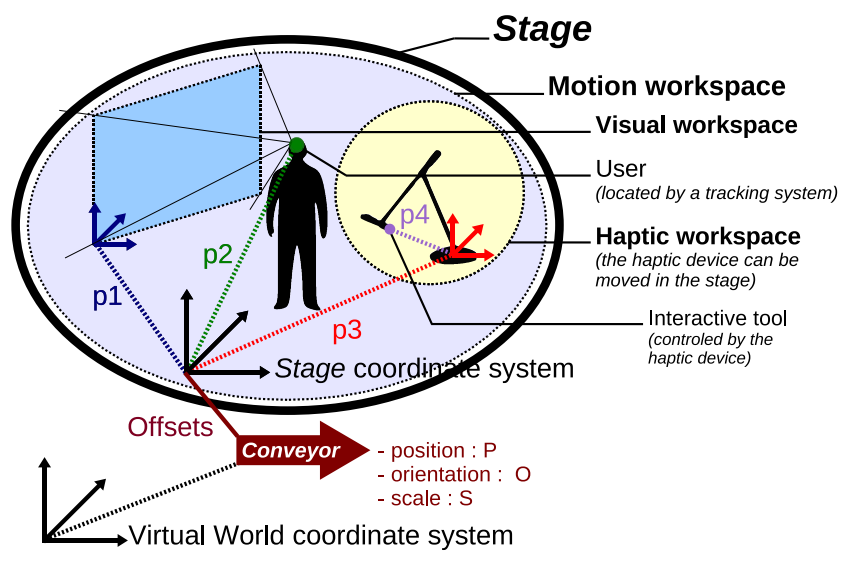

Fig. 1 The IIVC structure: the conveyor carries the stage with its workspaces in the virtual world.

by the features of the used physical devices. For example, motion workspace limits are often defined by the boundaries of the area in which users can move: position of the display devices of the immersive system, or limits of the tracking area. Each workspace has its own $3 \mathrm{D}$ shape and its own coordinate system to locate smaller workspaces or objects (real or virtual) that it contains.

- The stage is a virtual description of the users' real environment. It usually matches the room where users interact, but it is also the virtual space containing the virtual representations of users' workspaces. The user's workspaces are organized in a hierarchy of included 3D spaces into the stage. The stage uses its own coordinate system to locate directly or indirectly all the users' workspaces and all the objects of the IIVC. With this organization, the IIVC model is able to deal with physical reconfiguration such as modifications of workspace position and shape, additions of new screens or other devices, etc.

- The conveyor is the integration frame of the stage into the virtual world. This conveyor is located in the virtual world coordinate system, so it has its own position, orientation and scale in this world. The stage is linked to the conveyor with position, orientation, and scale offsets (see Figure 1). The conveyor also defines the navigation technique, the travel direction, the rotation center, and the scale of the IIVC. So the stage, its workspaces and consequently the objects inside the workspaces are carried by the conveyor when it moves or changes its scale in the virtual world.

The conveyor is totally virtual, while the stage makes the link between the real world and the virtual world. With this splitting into two parts, we propose to define the limit of the stage as the last physical level 
which cannot move during the simulation. For example, in a $\mathrm{CAVE}^{\mathrm{TM}}$, the limit of the stage will be the box defined by the screen's position.

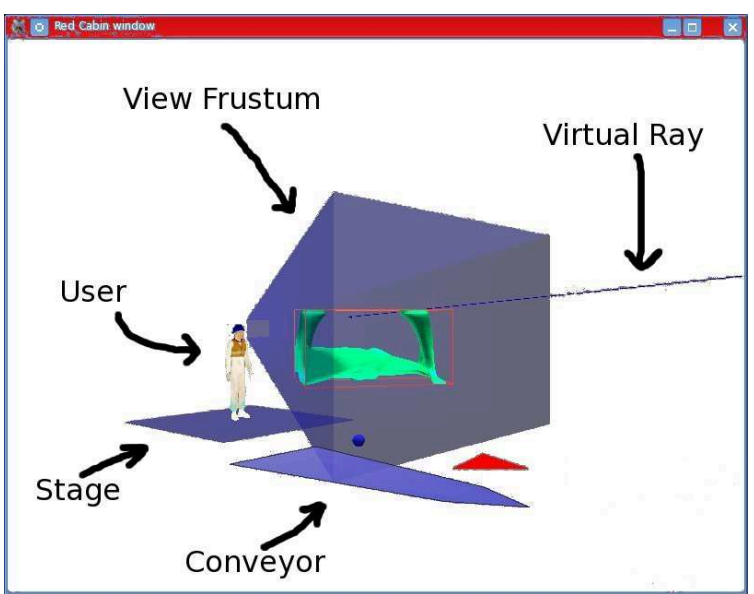

Fig. 2 One user within his/her IIVC viewed by another user: we can see him/her, his/her conveyor, his/her stage, his/her view frustum and his/her virtual ray.

Our model solves issues induced by collaborative sessions with remote users who interact from different physical environments: it enables users to perform a more effective collaboration by providing them a better understanding of the others' interaction capabilities, as it integrates users' physical workspaces and interaction tools in the virtual environment (see Figure 2).

\subsection{The IIVC Operators}

The operators are the basic and generic operations that are used to manage the IIVC structure. We provide a library that enables VR developers to implement several features for navigation and interaction.

First, the basic operators (Bo) are:

Bo1: modify the position (6 DoF) or scale of an object, Bo2: modify the features of an object (range of a virtual

light or a virtual ray, etc.),

Bo3: provide a new support to an object,

Bo4: modify the offset values of an object,

Bo5: add or remove an object into a workspace,

Bo6: provide a new conveyor to a stage,

Bo7: compute the local or global position of an object in relation to another frame.

Second, we provide higher level operators, called advanced operators (Ao), obtained through combination of basic operators, such as:

Ao1: superpose several stages or several conveyors,
Ao2: provide the same conveyor as a support to several stages,

Ao3: link a conveyor to an object,

Ao4: detect the proximity of objects,

Ao5: compute the intersection of workspaces,

Ao6: modify the shape of a workspace (for example the virtual frustum associated to a visual workspace),

Ao7: restrain DoF for position modification.

This set of seven high-level operators does not pretend to cover all possible operations in a VE, it will have to be extended in the future.

\section{Example of Design of a 3D CVE}

To illustrate the concepts of the IIVC model, let's consider the design of a collaborative application where two distant users will co-manipulate a virtual table through a 3-point manipulation technique.

In the example use-case scenario, one location is equipped with a large immersive system and a set of tracking devices, allowing for the user's head and two hands being tracked and used as an input modality for e.g. 3D cursors. The other location is equipped with only a desktop system and a $2 \mathrm{D}$ input device (such as a 2D mouse) used as an input modality for e.g. a 3D virtual ray, and a low-cost webcam-based head-tracking system used to change the user's point of view when he/she will move in front of his/her screen (see Figure 3 (b)).

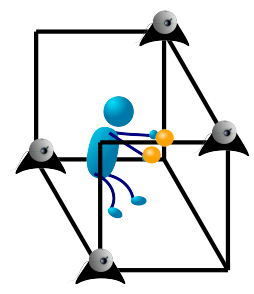

(a)



(b)
Fig. 3 (a) The first user in his/her large immersive system - (b) The second user with his/her desktop system

Indeed, the designer of such a distributed and multiuser application is facing several complex topics to meet its requirements: distribution and synchronization of the shared objects, adaptation of the rendering to asymmetric displays, adaptation of the interactions to asymmetric input devices, description of the interactions and providing of the good metaphor for co-manipulation. Here we will focus only on interaction and collaboration: the designer must describe both the interactive 
content of the virtual universe and the collaborative interaction tools that will be used to drive this content, through dedicated new collaboration metaphors able to provide the users with the best collaborative 3D interaction experience. Here, there will be a table, two 3D cursors, a 3D ray, an avatar of the position of the head of the first user, and an avatar of the position of the head of the second user. The $3 \mathrm{D}$ cursors and the $3 \mathrm{D}$ ray will make it possible to manipulate the table by using a 3-point manipulation technique [1].

Although it is important to work on abstract interaction and metaphors, sometimes the hardware features of the real environment of the users must be embedded in the run-time VR application to take into account the physical environment of the users, such as the size and the resolution of the screens of a VR immersive system, as recommended by the IIVC model. So, this designer also should be able to integrate a representation of the run-time hardware components in the virtual environment, to make the users aware of the limitations of these components. According to the IIVC model, it leads to the creation of new virtual objects that must be added to the CVE, corresponding to the users' workspaces: a representation of the bounds of the tracking systems of each user, a representation of the display surfaces of the first user, and a representation of the field of view of the second user, as illustrated in Figure 4.

As an immediate result, providing a $3 \mathrm{D}$ model of the physical environment and embedding it into the virtual environment through the IIVC model can prevent the user from hurting himself. Indeed, by tracking the user and through the knowledge of the position of the real objects surrounding him/her, the IIVC can compute the distance between the user and the real objects (co-localized with their virtual representations) and provide some warnings to inform the user of his/her proximity to the real objects, especially those that are difficult to perceive such as the display screens of an immersive system, or those that cannot be perceived because the user is using a HMD. Figure 5 illustrates how it is possible to show the location of the display screens of an immersive display to an immersed user in order to prevent him/her from hitting the walls: when the user stands far enough from the walls, the virtual representations of the screens are totally transparent, then when the user comes closer to them they become visible, last when the user comes dangerously closer to the screens they become totally opaque and we provide additional feedback that can be also visual or can use another mode of communication (for example we can use sound feedback).

Nevertheless, in some cases this solution must be enhanced, for example when other $3 \mathrm{D}$ virtual objects of the virtual environment occlude the virtual objects that represent the screens. To cope with these potential occlusions, we have extended the visual effect to the whole volume of the stage, as illustrated in figure 6 .

\section{Providing cognitive cues without co-localization}

As high-quality immersive systems are very expensive, collaborative virtual environments sometimes use asymmetrical configurations, and users can join collaborative sessions through simple workstations. Most of the time the actions of these users cannot be tracked so it is not possible to establish a good co-localization between their gestures and the virtual tools they are manipulating. It is also difficult to use efficiently the classical $3 \mathrm{D}$ interaction metaphors. In such conditions, basic 2D interaction tools such as a $2 \mathrm{D}$ pointer driven with a classical 2D mouse can be as efficient as the usual 3D metaphors for simple tasks such as object selection and manipulation (3D positioning, for instance).

The main problem when using such basic $2 \mathrm{D}$ interaction metaphors is that it is difficult to make a user aware of the interactions of the other users who are using classical 2D interaction tools because these $2 \mathrm{D}$ tools are not associated with any $3 \mathrm{D}$ virtual objects.

Our idea is to use a $3 \mathrm{D}$ virtual ray [38] that could be as easy to drive as a classical $2 \mathrm{D}$ mouse pointer. This is the reason why we have proposed a new pointer that looks like a $2 \mathrm{D}$ pointer but that can be associated with a $3 \mathrm{D}$ geometry in order to visually appear within a CVE, we call it the 2D Pointer / 3D Ray [16]. This $2 \mathrm{D}$ pointer can be used like a classical $2 \mathrm{D}$ pointer and can be driven by any device that can control a $2 \mathrm{D}$ position: a classical 2D mouse, a gamepad, or a Nintendo wiimote remote gaming controller. The 3D geometry of this pointer will be a virtual ray, so other users can be easily made aware of the movement of this $3 \mathrm{D}$ ray, in the same way they can be made aware of the evolution of classical 3D interaction metaphors. This 2D Pointer / 3D Ray will use the classical ray-casting technique for object selection and manipulation. In this way, its behavior is similar to the aperture-based selection technique [22] and to the technique developped in [44].

The result looks like a classical $2 \mathrm{D}$ pointer moving on the surface of the screen. In fact it is a quite thin and long 3D virtual ray, moving very near the user's viewpoint in order to avoid being occluded by objects of the VE, staying always at the same depth. The orientation of this $3 \mathrm{D}$ virtual ray is calculated in a way that its projection on the screen is always a small spot, as illustrated in Figure 7. 


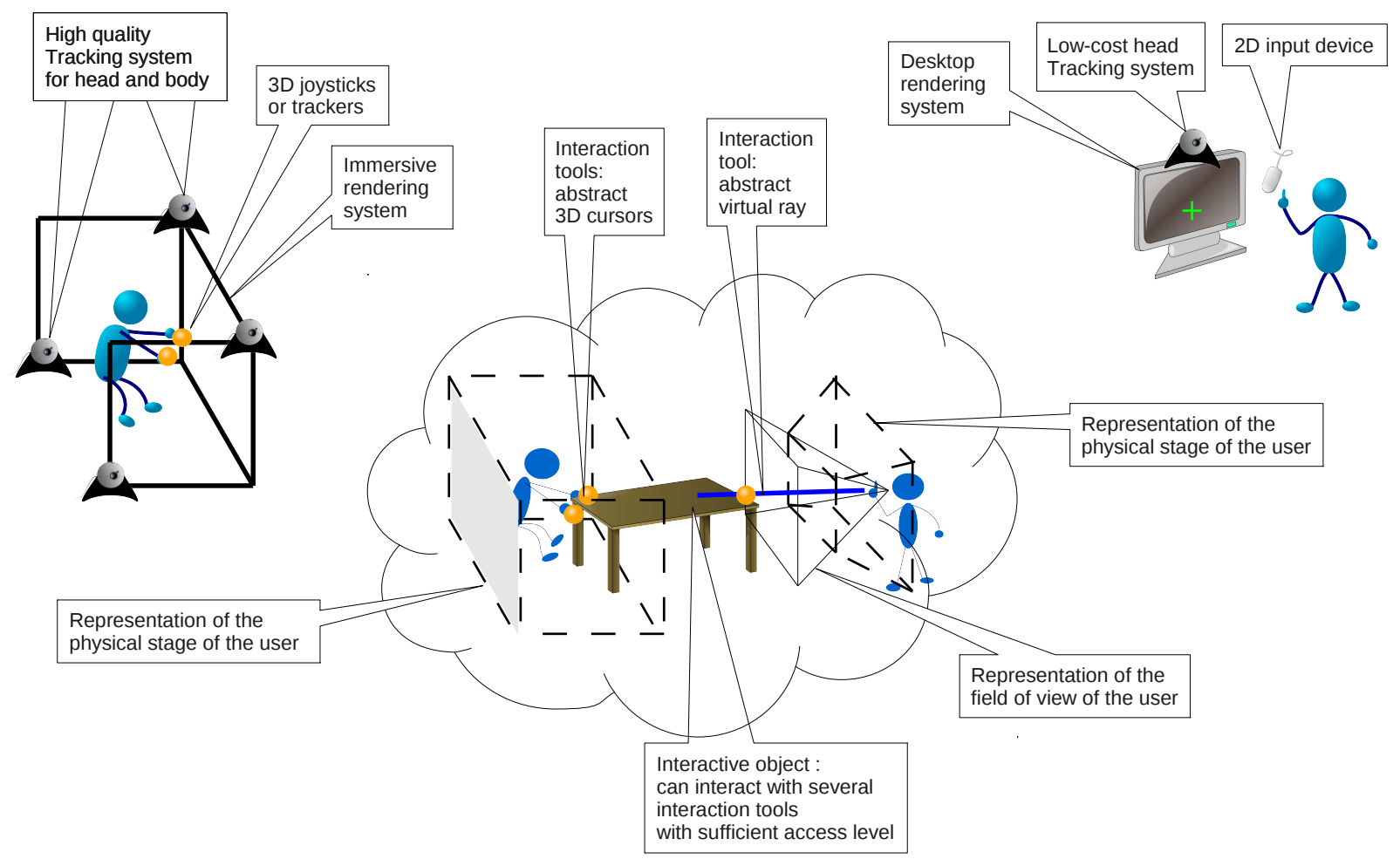

Fig. 4 Modeling collaborative interaction in a CVE: the need to embed in the virtual world the physical features of the real environments that surround the users
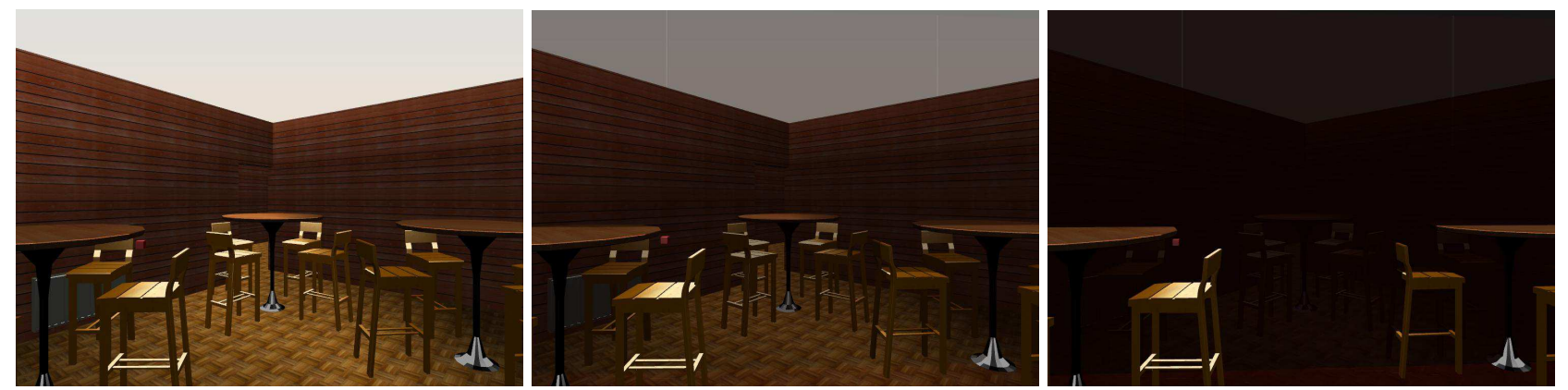

Fig. 5 Showing the location of the display screens of an immersive display to an immersed user to prevent him/her from hitting the walls. From the image on the left to the image on the right, the 3D visualization of the front screen evolves from being totally transparent to only $30 \%$ transparent as the user approaches with his/her hand (represented by the small red cube). The objects placed in front of the screen are not affected by this change, it makes the user aware of which objects can be reached safely by a physical move of his/her hand.

As shown in Figure 7, the 2D device used to control the pointer will provide the $X c$ and the $Y c$ values, and the $Z c$ value is a chosen one, so the rho and theta values can be calculated this way, if the rho angle (the heading) is first applied around the $Y$ axis and then the theta angle (the elevation) is applied around the $X^{\prime}$ axis :

$-r h o=\operatorname{atan}(-X c / Z c)$

- theta $=\operatorname{atan}(Y c / \operatorname{sqrt}(X c * X c+Z c * Z c))$

This way, the user of the 2D Pointer / 3D Ray will be using a $3 \mathrm{D}$ virtual ray that will behave like a $2 \mathrm{D}$ pointer (see Figure 8(a)), while other users will see a
$3 \mathrm{D}$ virtual ray moving thanks to the action of the first user (see Figure 8(b)).

This 2D Pointer / 3D Ray is a new metaphor for 3D interaction within Collaborative Virtual Environments: the 2D Pointer / 3D Ray, which associates a 3D representation with a $2 \mathrm{D}$ pointing device (for example a $2 \mathrm{D}$ mouse). This metaphor allows an asymmetric collaboration between users immersed within a CVE (via stereovision and head-tracking) and users simply sitting in front of the screen of their workstation. The user without immersion will interact as easily as if he/she had a simple $2 \mathrm{D}$ pointer, as the associated $3 \mathrm{D}$ ray (a $3 \mathrm{D}$ 

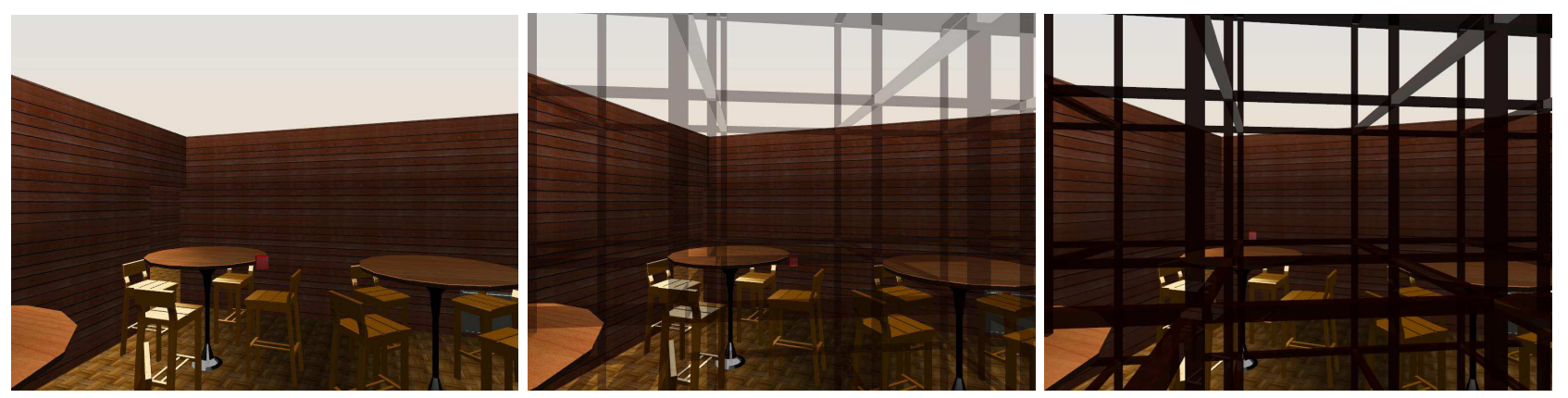

Fig. 6 Alternative way of showing to the user the limits of his/her physical environment, to prevent him/her from hitting the walls. From the image on the left to the image on the right, a 3D visualization of the whole physical space (a 3D grid) evolves from transparent to only $30 \%$ transparent as the user approaches the screen with his/her hand (represented by the small red cube).

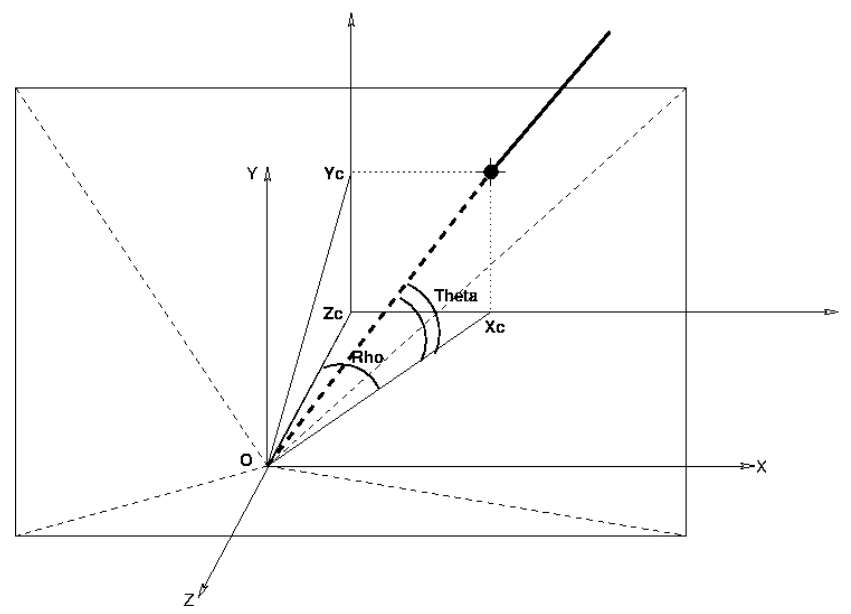

Fig. 7 Projection of the 3D Ray as a small spot on the screen.

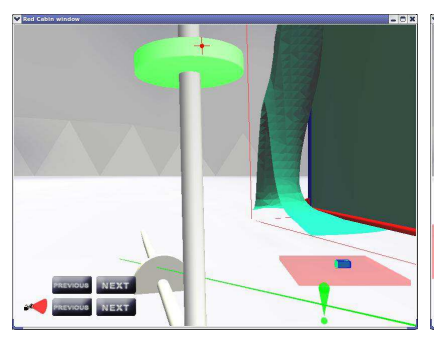

(a)

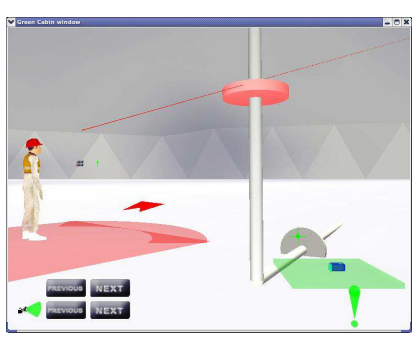

(b)
Fig. 8 (a) On the left, user 1 moves a 3D slider with his/her red $2 \mathrm{D}$ pointer and he/she sees the green $3 \mathrm{D}$ virtual ray of user 2 ready to select another slider. (b) On the right, user 2 is ready to select a slider with his/her green $2 \mathrm{D}$ pointer while he/she is looking at user 1 moving a slider with his/her red $3 \mathrm{D}$ virtual ray.

virtual ray) will be continuously moved and oriented in a way that its projection on the screen of the user will always be a small spot. The other users of the CVE will be made aware of the action of this user thanks to the movements of his/her associated $3 \mathrm{D}$ virtual ray.

\section{Implementation example}

Collaboration can provide a powerful technique for exploration of large unknown virtual environments. It makes it possible to support exploring users to deal with lack of spatial knowledge. Although CVEs have been developed to provide a framework of information sharing and communication [30][14][11], collaborative navigation task in such environments has not been largely explored and only limited attention has been devoted to evaluate its efficiency in navigation in VEs.

It is essential for navigation in a CVE to support communication between users because it is vital to understand what the others are referring to. So the communication technique for collaboration, especially for navigation in CVEs, should be simple, intuitive, efficient and non-verbal (because users do not always speak the same language). Based upon these points, our primary motive was to develop guiding techniques enabling helping users to guide an exploring user toward target places in complex large-scale CVEs. We share this objective with the organizers of the 3DUI Contest 2012 and its participants. As navigation aids, some techniques had been proposed such as "anchors" and a string of blue arrows that connects them or directional arrows [2][34], point light sources [10] or light signal or beacons [35][34][43].

We have developed three guiding techniques in the form of navigation aids (arrows, light source and compass) that would enable one or several helping user(s) to guide an exploring user who is traveling in an unfamiliar 3D VE efficiently. We have also proposed a fourth solution that remotely takes the control of a user's viewpoint. The implementation of these guiding techniques relies strongly on the IIVC model. A complete description of these navigation aids has been detailed in [33]. 


\subsection{Arrows}

The first guiding technique is based on directional arrows (see Figure 9) that are drawn in the view of the helping users to indicate the direction or the path that the exploring user has to follow. The helping users can draw as many directional arrows of different sizes as they want. However, so many directional arrows added within the environment or too big arrows may affect the immersion of the exploring user. As a result, the helping users have to determine when, how and where to put directional arrows to guide efficiently the exploring user. These arrows will disappear after a while. So the helping users are recommended to draw directional arrows within the exploring user's visibility zone.

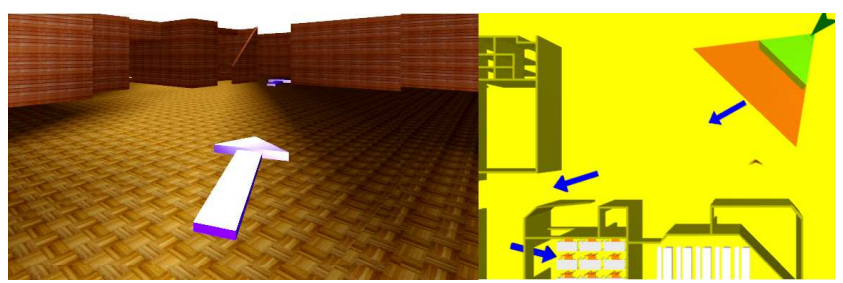

Fig. 9 Directional arrows in the exploring user's and the helping user's views.

\subsection{Light source}

The second guiding technique is based on a light source used as a beacon to light up a path to target places (see Figure 10). The exploring user cannot see the light source itself but only its effect on objects within the environment. The light source is attached to a support (a 3D object) that can only be seen by a helping user. This helping user controls the light source by moving its support with a $3 \mathrm{D}$ cursor and shows up the path the exploring user must follow. It is important for the helping user to estimate if the exploring user can perceive the effect of the light on the environment.

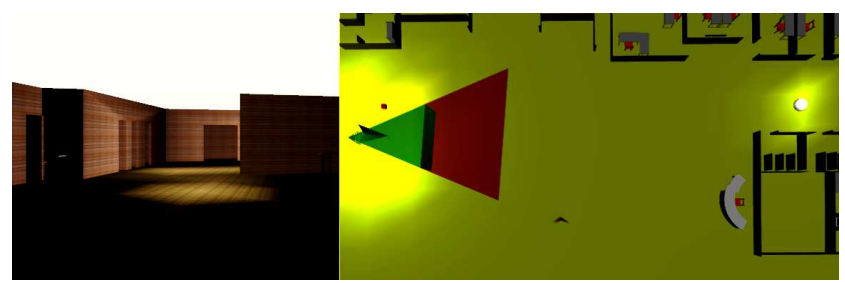

Fig. 10 Light source in the exploring user's and the helping user's views.

\subsection{Compass}

The third guiding technique is based on a compass attached to the position of the exploring user (with an offset), a typical tool to navigate in VEs (see Figure 11). The compass does not point directly to a target location, but points to the location of another virtual object that plays the role of the "north" of this compass, and this object cannot be seen by the exploring user. A helping user can control this "north" by moving it with a 3D cursor, to show up to the exploring user the path he/she must follow. So by moving the "north" of the compass, a helping user can guide the exploring user to pass across hallways, rooms, doors, etc. before reaching the target location. It is thus a simple and powerful tool to guide the exploring user in any VE.

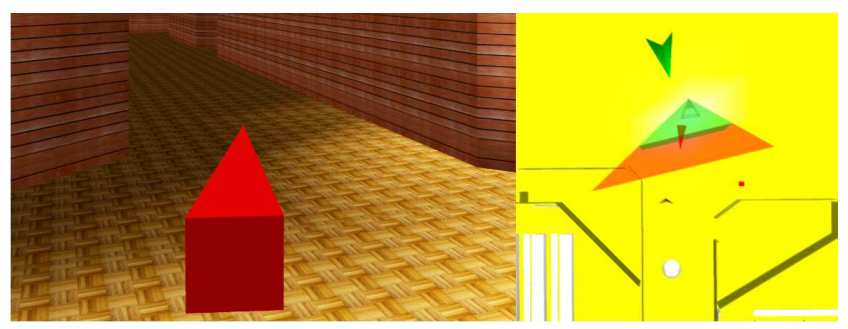

Fig. 11 Compass in the exploring user's and the helping user's views.

6.4 Remotely controlling the position of the main user

Our last proposition is to allow the helping user to grab the main user to bring him/her near his/her next target location. This grabbing is very easy to implement through our IIVC model of the exploring user's environment: the helping user has only to interact with the conveyor of the exploring user. This technique can be interesting when the main user moves too slowly or when he/she badly understands the indications given by the helping user. During this particular guiding, the main user is made aware of the guiding through a semitransparent object that is surrounding him/her, inviting him/her not to move during the guiding, as illustrated in Figure 12.

\subsection{The guiding viewpoints}

To be able to use these guiding techniques in an efficient way, we built two principal kinds of views for helping users: a bird's eye view (see the parts on the right of Figures 9, 10 and 11) and a first-person perspective by "looking over the exploring user's shoulder" (just like a 



Fig. 12 The main user surrounded by a blue grid inviting him/her to stay immobile during the remote guiding. The image on the left shows the view of the main user, while the image on the right shows the view of the guiding user.

camera attached to the shoulder of the exploring user) (see Figure 13). The bird's eye view can be considered as a 3D map or a World-In-Miniature [42]. To be efficiently used, these views must contain the $3 \mathrm{D}$ representation of some physical features of the exploring user's stage, especially the position of his/her head which is used to build his/her virtual field of view. All these features are modeled according to our IIVC structure. The representation of this field of view is dynamically reshaped while the user is moving inside his/her stage. Additionally, as this stage is attached to the virtual conveyor used for navigation, this stage moves with the conveyor and brings along the virtual field of view of the exploring user.



Fig. 13 A "looking over the exploring user's shoulder" view of the helping user.

\subsection{Asymmetrical collaborative navigation}

The propositions we have made for guiding an exploring user immersed in a 3D CVE use an asymmetrical collaborative configuration for an exploring user and helping users. Usually, asymmetrical collaborations can be imposed by some materials limitations of one or several users. In most cases, only one user (the exploring user) benefits from a fully immersive system. This is why we have provided helping users with some interesting viewpoints (complementary to the viewpoint of the exploring user) and guiding metaphors, even if these helping users do not benefit from a fully immersive system.

If helping users could also benefit from a full immersion in the virtual environment, we could implement our metaphors by providing the "over the exploring user's shoulder" view as the main view of helping users, and by replacing the bird's eye view by a 3D World In Miniature (WIM). This WIM would be embedded in helping users' IIVC. For example, they could interact through objects in the WIM to guide the exploring user: by tracing directional arrows in the WIM; by manipulating the miniature of the light; by manipulating the miniature of "north" of the compass; or by moving the miniature of the exploring user's conveyor.

\section{Conclusion and perspectives}

In this paper we have explained why most Virtual Reality (VR) systems must consider the users' physical environment to immerse these users in a virtual world and to make them aware of their interaction capabilities. This avoids disturbing users' interactions with their physical environment that would alter their feeling of presence. We propose to use the IIVC model that enables VR developers to embed the users' physical environment into the Virtual Environment (VE) when designing new applications, especially collaborative ones. Moreover, the 3D representation of these physical features can be useful for collaboration between distant users because they can make a user aware of the physical limitations of the others he/she is collaborating with. We have explained how to extract the physical features of the real environment of a user in order to embed them in the $\mathrm{VE}$ and we have illustrated how these features can be used for a collaborative navigation task.

All these features are implemented in the Collaviz framework [15] dedicated to Collaborative Virtual Reality, which is available for all partners of the VISION$\mathrm{AIR}^{1}$ project. Its implementation of the IIVC model makes it easy to install in any immersive system of this project, even if the partners prefer to use a specific 3D API for rendering, as its architecture makes it possible to use several rendering engines [17]. Furthermore, its collaborative capabilities [20] make it possible to deploy collaborative applications between several VISIONAIR distant sites. Collaborative experiments have already been conducted between IRISA/INRIA Rennes (France) and University College of London (UCL) (England) [21].

\footnotetext{
1 http://www.infra-visionair.eu/
} 
Frameworks for collaborative virtual environments are now mature enough to allow researchers to focus on higher-level description of collaboration rather than on low-level system features. Establishing as automatically as possible a good matching between the virtual environment and the physical environment of the endusers is still a challenge and our IIVC concept can be an answer to this problem. However, describing all the components of an instance of the IIVC is still a consequent programming work, and a Model Driven Engineering (MDE) [27] approach would certainly be very useful, with a Domain Description language (DSL) for describing the features of the physical environment and their matching with objects of the virtual universe.

We still have to improve the collaboration between distant users who are sharing a virtual environment, by proposing more efficient metaphors for 3D collaborative interactions. This topic is still very relevant: it was the main subject of the 3DUI 2012 contest. We participated to this contest by proposing some solutions based on the Collaviz framework in order to enhance collaboration between two users [34] and we will go on proposing new solutions dedicated to fully immersive collaborations.

At last, we plan to use such collaborative framework to animate collaborative ergonomics design sessions by embedding a symbolic 3D avatar of a user in the framework and share it with the other users. The idea is to compute and visualize bio-mechanical risk factors involved in musculo-skeletal disorder appearance at work (e.g. posture scores, kinematic features, muscle forces, ... ) [36]. The worker (main user) will be immersed in a virtual representation of the workstation while ergonomists (collaborators) see in real-time biomechanical factors during the realization of work tasks. Collaborators then will be able to interactively propose enhancements to the main user in the way he/she performs the task (more comfortable postures, less awkward motions) or to modify the working environment (features position, work cycle organization, etc.). Some metaphors inspired from the guiding techniques we presented in part 6 could be designed and tested for such purpose. Prior to the use of such tool, a qualification of the framework and the simulator in terms of fidelity is warranted [37]. Such use could be extended to several other activities, such as coaching, training or reeducation.

\section{Acknowledgment}

We thank Foundation Rennes 1 "Progress, Innovation, Entrepreneurship" and the European Project VISIONAIR (grant agreement 262044) for their support.

\section{References}

1. Aguerreche, L., Duval, T., Lécuyer, A.: 3-Hand Manipulation of Virtual Objects. In: Proceedings of JVRC (Joint Virtual Reality Conference of EGVE - ICAT - EuroVR), pp. 153-156 (2009)

2. Bacim, F., Ragan, E.D., Stinson, C., Scerbo, S., Bowman, D.A.: Collaborative navigation in virtual search and rescue. In: Proceedings of IEEE Symposium on 3D User Interfaces, pp. 187-188. IEEE Computer Society (2012)

3. Baranyi, P., Csapo, A.: Definition and synergies of cognitive infocommunications. Acta Polytechnica Hungarica $\mathbf{9}(1), 67-83$ (2012)

4. Benford, S., Bowers, J., Fahlén, L.E., Greenhalgh, C.: Managing Mutual Awareness in Collaborative Virtual Environments. In: Proceedings of VRST, pp. 223-236 (1994)

5. Benford, S., Greenhalgh, C., Rodden, T., Pycock, J.: Collaborative Virtual Environments. Communication of the ACM 44(7), 79-85 (2001). DOI http://doi.acm.org/10.1145/379300.379322

6. Bowman, D., Coquillart, S., Froehlich, B., Hirose, M., Kitamura, Y., Kiyokawa, K., Stuerzlinger, W.: 3D User Interfaces: New Directions and Perspectives. Computer Graphics and Applications, IEEE 28(6), 20-36 (2008)

7. Bowman, D.A., Johnson, D.B., Hodges, L.F.: Testbed evaluation of virtual environment interaction techniques. In: Proceedings of the ACM symposium on Virtual reality software and technology, VRST '99, pp. 26-33. ACM, New York, NY, USA (1999)

8. Bowman, D.A., Kruijff, E., LaViola, J.J., Poupyrev, I.: 3D User Interfaces: Theory and Practice. Addison Wesley (2004)

9. Butterworth, J., Davidson, A., Hench, S., Olano, M.T.: 3DM: A Three Dimensional Modeler using a HeadMounted Display. In: Proceedings of the Symposium on Interactive 3D graphics, pp. 135-138 (1992)

10. Cabral, M., Roque, G., Santos, D., Paulucci, L., Zuffo, M.: Point and go: Exploring 3d virtual environments. In: Proceedings of IEEE Symposium on 3D User Interfaces, pp. 183-184. IEEE Computer Society (2012)

11. Churchill, E.F., Snowdon, D.N., Munro, A.J.: Collaborative Virtual Environments: Digital Places and Spaces for Interaction. Springer Verlag London Ltd, London, United Kingdom (2001)

12. Cirio, G., Marchal, M., Regia-Corte, T., Lécuyer, A.: The Magic Barrier Tape: A Novel Metaphor for Infinite Navigation in Virtual Worlds with a Restricted Walking Workspace. In: Proceedings of VRST, pp. 155-162 (2009)

13. Cruz-Neira, C., Sandin, D.J., DeFanti, T.A.: Surroundscreen projection-based virtual reality: the design and implementation of the cave. In: Proceedings of SIGGRAPH'93, pp. 135-142. ACM, New York, NY, USA (1993)

14. Dumas, C., Degrande, S., Saugis, G., Chaillou, C., Viaud, M.L., Plenacoste, P.: Spin: a 3d interface for cooperative work. Virtual Reality Society Journal (1999)

15. Dupont, F., Duval, T., Fleury, C., Forest, J., Gouranton, V., Lando, P., Laurent, T., Lavoué, G., Schmutz, A.: Collaborative Scientific Visualization: The COLLAVIZ Framework. In: JVRC Demos (2010)

16. Duval, T., Fleury, C.: An asymmetric $2 \mathrm{~d}$ pointer/3d ray for $3 \mathrm{~d}$ interaction within collaborative virtual environments. In: Web3D'09: Proceedings of the 14th International Conference on 3D Web Technology, pp. 33-41. ACM, New York, NY, USA (2009) 
17. Duval, T., Fleury, C.: Collaboration between networked heterogeneous $3 \mathrm{~d}$ viewers through a pac-c3d modeling of the shared virtual environment. In: Proceedings of ICAT (2011)

18. Duval, T., Nguyen, T.T.H., Fleury, C., Chauffaut, A., Dumont, G., Gouranton, V.: Embedding the Features of the Users' Physical Environments to Improve the Feeling of Presence in Collaborative Virtual Environments. In: CogInfoCom 2012 (3rd IEEE International Conference on Cognitive Infocommunications), pp. 243-248. Kosice, Slovakia (2012)

19. Fleury, C., Chauffaut, A., Duval, T., Gouranton, V., Arnaldi, B.: A Generic Model for Embedding Users' Physical Workspaces into Multi-Scale Collaborative Virtual Environments. In: Proceedings of ICAT, pp. 1-8 (2011)

20. Fleury, C., Duval, T., Gouranton, V., Arnaldi, B.: A New Adaptive Data Distribution Model for Consistency Maintenance in Collaborative Virtual Environments. In: Proceedings of JVRC, pp. 29-36 (2010)

21. Fleury, C., Duval, T., Gouranton, V., Steed, A.: Evaluation of Remote Collaborative Manipulation for Scientific Data Analysis. In: Proceedings of VRST. ACM, Toronto, Canada (2012)

22. Forsberg, A., Herndon, K., Zeleznik, R.: Aperture based selection for immersive virtual environments. In: UIST '96: Proceedings of the 9th annual ACM symposium on User interface software and technology, pp. 95-96. ACM, New York, NY, USA (1996). DOI http://doi.acm.org/10.1145/237091.237105

23. Fraser, M., Benford, S., Hindmarsh, J., Heathq, C.: Supporting Awareness and Interaction through Collaborative Virtual Interfaces. In: Proceedings of UIST, pp. 27-36 (1999)

24. Frécon, E., Nöu, A.A.: Building distributed virtual environments to support collaborative work. In: Proceedings of the ACM symposium on Virtual reality software and technology, VRST '98, pp. 105-113. ACM, New York, NY, USA (1998)

25. Galambos, P., Baranyi, P.: Virca as virtual intelligent space for rt-middleware. In: Advanced Intelligent Mechatronics (AIM), 2011 IEEE/ASME International Conference on, pp. 140-145 (2011)

26. Hand, C.: A Survey of 3D Interaction Techniques. Computer Graphics Forum 16(5), 269-281 (1997)

27. Jézéquel, J.M., Barais, O., Fleurey, F.: Model Driven Language Engineering with Kermeta. In: 3rd Summer School on Generative and Transformational Techniques in Software Engineering. LNCS 6491, Springer (2010)

28. Lalioti, V., Garcia, C., Hasenbrink, F.: Virtual meeting in cyberstage. In: Proceedings of the ACM VRST, pp. 205-212. ACM, New York, NY, USA (1998)

29. Leigh, J., Johnson, A., Vasilakis, C., DeFanti, T.: Multiperspective Collaborative Design in Persistent Networked Virtual Environments. In: Proceedings of VRAIS'96, pp. 253-260 (1996)

30. Macedonia, M., Zyda, M.J., Pratt, D., Barham, P., Zeswitz, S.: Npsnet: A network software architecture for large scale virtual environments. Presence 3(4), 265-287 (1994)

31. Margery, D., Arnaldi, B., Plouzeau, N.: A General Framework for Cooperative Manipulation in Virtual Environments. In: Virtual Environments'99, pp. 169-178 (1999)

32. Mulder, J.D., Boschker, B.R.: A Modular System for Collaborative Desktop VR/AR with a Shared Workspace. Proceedings of the IEEE Virtual Reality Conference 0, 75 (2004)
33. Nguyen, T.T.H., Duval, T., Fleury, C.: Guiding Techniques for Collaborative Exploration in Multi-Scale Shared Virtual Environments. In: GRAPP International Conference on Computer Graphics Theory and Applications, pp. 327-336. Barcelone, Spain (2013). URL http://hal.inria.fr/hal-00755313

34. Nguyen, T.T.H., Fleury, C., Duval, T.: Collaborative Exploration in a Multi-Scale Shared Virtual Environment. In: Proceedings of IEEE Symposium on 3D User Interfaces, pp. 181-182. IEEE Computer Society (2012)

35. Notelaers, S., Weyer, T.D., Goorts, P., Maesen, S.: Heatmeup: a 3dui serious game to explore collaborative wayfinding. In: Proceedings of IEEE Symposium on 3D User Interfaces, pp. 177-178. IEEE Computer Society (2012)

36. Pontonnier, C., Dumont, G.: From Motion Capture to Muscle Forces in the Human Elbow Aimed at Improving the Ergonomics of Workstations. Virtual and Physical Prototyping 5, 113-122 (2010)

37. Pontonnier, C., Dumont., G., Samani, A., Madeleine, P., Badawi, M.: Designing and evaluating a workstation in real and virtual environment: from digital mock-up to realization. In: 3rd IEEE International Conference on Cognitive Infocommunications (CogInfoCom 2012) (2012)

38. Poupyrev, I., Weghorst, S., Billinghurst, M., Ichikawa, T.: Egocentric Object Manipulation in Virtual Environments: Empirical Evaluation of Interaction Techniques. Computer Graphics Forum 17(3) (1998). URL citeseer.ist.psu.edu/poupyrev98egocentric.html

39. Razzaque, S.: Redirected Walking. Ph.D. thesis, University of North Carolina at Chapel Hill (2005)

40. Ruddle, R.A., Savage, J.C.D., Jones, D.M.: Symmetric and Asymmetric Action Integration during Cooperative Object Manipulation in Virtual Environments. ACM Transactions on ComputerHuman Interaction 9(4), 285-308 (2002). DOI http://doi.acm.org/10.1145/586081.586084

41. Smets, G.J.F., Stappers, P.J., Overbeeke, K.: Designing in virtual reality: implementing perception-action coupling with affordances. In: Proceedings of ACM VRST, pp. 97-110. World Scientific Publishing Co., Inc., River Edge, NJ, USA (1994)

42. Stoakley, R., Conway, M.J., Pausch, R.: Virtual reality on a wim: Interactive worlds in miniature. In: Proceedings of the SIGCHI conference on Human Factors in Computing Systems, pp. 265-272. ACM Press/Addison-Wesley Publishing Co. New York (1995)

43. Wang, J., Budhiraja, R., Leach, O., Clifford, R., Matsua, D.: Escape from meadwyn 4: A cross-platform environment for collaborative navigation tasks. In: Proceedings of IEEE Symposium on 3D User Interfaces, pp. 179-180. IEEE Computer Society (2012)

44. Ware, C., Lowther, K.: Selection Using a One-eyed Cursor in a Fish Tank VR Environment. ACM Trans. Comput.-Hum. Interact. 4(4), 309-322 (1997). DOI http://doi.acm.org/10.1145/267135.267136

45. Williams, B., Narasimham, G., Rump, B., McNamara, T.P., Carr, T.H., Rieser, J., Bodenheimer, B.: Exploring Large Virtual Environments with an HMD When Physical Space is Limited. In: Proceedings of the 4th Symp. on Applied perception in graphics and visualization, pp. 41-48 (2007)

46. Zahorik, P., Jenison, R.L.: Presence as being-in-theworld. Presence: Teleoperators and Virtual Environments $\mathbf{7}(1), 78-89$ (1998) 